\title{
Capturing the Structure of Mesoporous Silica Nanoparticles in Solution With Cryo-TEM
}

\author{
Katherine A. Spoth ${ }^{1}$, Yao Sun ${ }^{2}$, Ulrich Wiesner ${ }^{2}$, Lena F. Kourkoutis ${ }^{1,3}$ \\ 1. Department of Applied and Engineering Physics, Cornell University, Ithaca, NY 14853, USA. \\ 2. Department of Materials Science, Cornell University, Ithaca, NY 14853, USA. \\ 3. Kavli Institute for Nanoscale Science, Cornell University, Ithaca, NY 14853, USA.
}

Ordered mesoporous materials have attracted great interest due to their potential in energy applications. The materials' large surface area and interconnected ordered pore structure make them good candidates for electrode materials in fuel cells and batteries, and for applications in photovoltaics [1-2]. While their synthesis is performed in solution, TEM characterization of mesoporous materials is almost exclusively reported after drying. The drying process itself can, however, result in structural modifications or even total collapse. Advancement in the fundamental understanding of the formation process of these mesoporous structures and their control requires imaging of the materials' native structure in solution.

Here, we use cryo-TEM to image mesoporous silica nanoparticles (MSNs) snap-frozen in solution and reveal a $10 \%$ reduction in the lattice constant of the ordered pore structure upon drying. While nanometer and atomic resolution imaging of nanoparticles in liquids has been demonstrated, the inherently dynamic nature of liquid cell TEM prevents high-resolution chemical and three-dimensional structural characterization. In cryo-TEM imaging, samples are immobilized through vitrification of the surrounding liquid allowing direct observation of samples in solution at high resolution.

Samples for cryo-TEM were prepared through plunge freezing, in which a drop of MSNs in solution was applied to a Quantifoil holey carbon TEM grid, blotted, and plunged into a mixture of condensed ethane and propane, vitrifying the liquid. Samples were imaged at $-174^{\circ} \mathrm{C}$ using a Gatan 626 cryo-transfer holder which allows transfer of samples under liquid nitrogen, essential to prevent warming and recrystallization of the solution. A vitrified sample imaged by cryo-TEM is shown in Fig. 1(a); holes in the carbon film are filled with vitrified solution and suspended nanoparticles. Figure 1(b) shows MSNs embedded in vitrified ice at the edge of a hole. For images in dry condition, particles in solution were dried in low humidity and imaged at $19^{\circ} \mathrm{C}$. Images were acquired using a FEI Tecnai T12 BioTwin.

MSNs in solvents of water and ethanol were imaged in solution and in dry condition. The star shape observed for MSNs in water by cryo-TEM, Fig. 1(c), is consistent with the shape of dried samples, Fig. 1(d). In contrast, samples in ethanol show a symmetric hexagonal structure (in solution Fig. 1(e), dried Fig. 1(f)). The presence of water causes the condensation of the MSN matrix to silica, which drives the structural transformation. While the external shape of the particles in ethanol is hexagonal in both dried and "wet" conditions, FFTs taken from the individual MSNs indicate a 10\% reduction in the pore lattice spacing upon drying from ethanol. Cryo-TEM is unique in allowing the direct observation of structures in solution and could advance the understanding of MSN formation that in turn would allow for control over the structure of MSNs to favorably tune their properties for energy applications [3].

[1] Y Ye et al, Nanoscale, 5 (2013), p. 4584.

[2] F Hoffmann et al, Angew. Chem. Int. Ed. 45 (2006), p. 3216 - 3251.

[3] The CCMR EM Facility is supported through the NSF MRSEC program (DMR-1120296). 

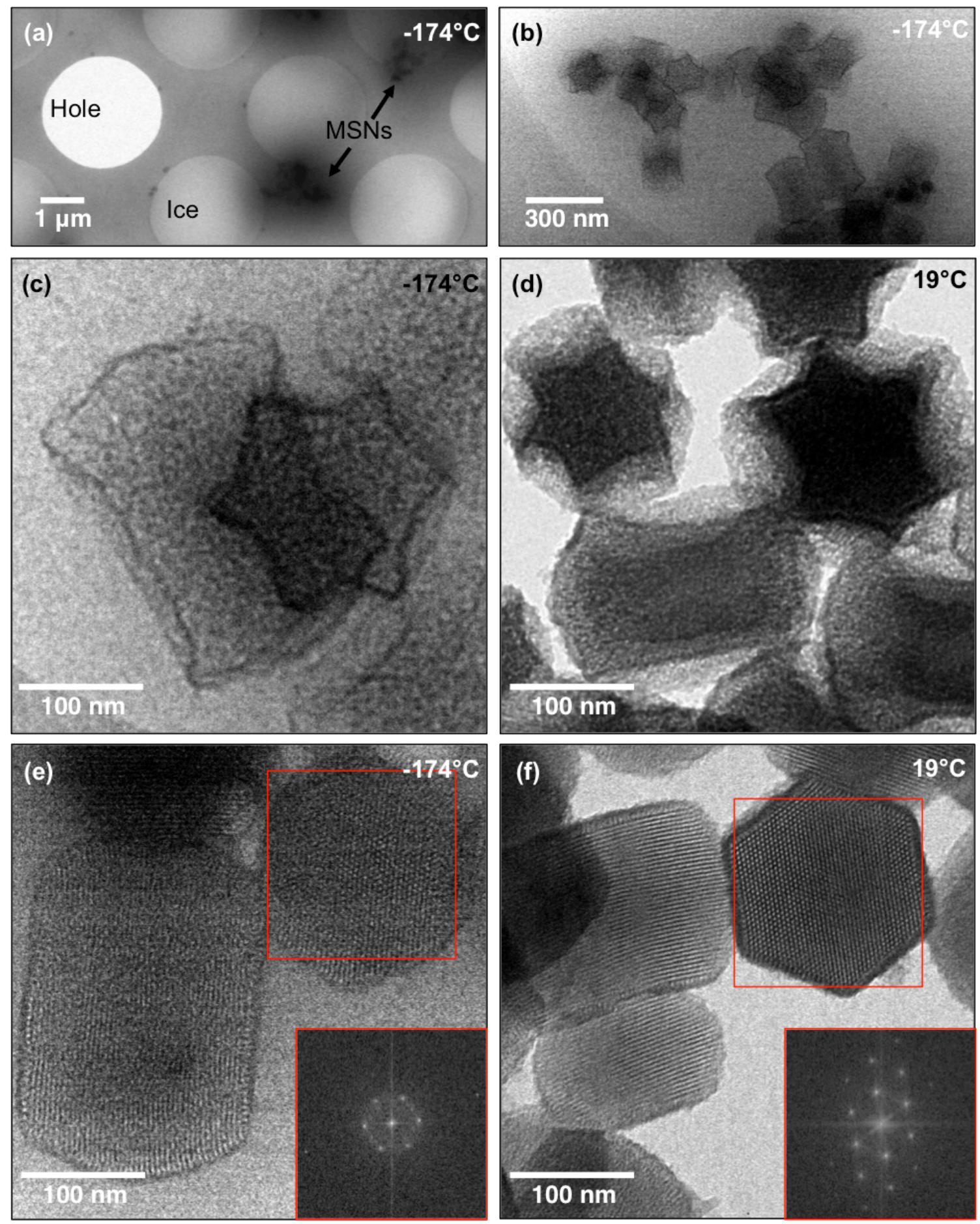

Figure 1. TEM images of MSNs snap-frozen in solution ("wet") and dried. (a) Cryo-TEM (-174 $\left.{ }^{\circ} \mathrm{C}\right)$ image of nanoparticles in water. Vitrified liquid suspends sample and fills majority of holes in carbon support film. (b)-(d) MSNs in water showing star shape in "wet" (b), (c), and dry conditions (d). (e), (f) MSNs in ethanol with hexagonal shape; FFTs from areas indicated suggest that the particles' pore lattice shrinks by $10 \%$ upon drying. 